\title{
Recent advancements in erythrocytes, platelets, and albumin as delivery systems
}

This article was published in the following Dove Press journal:

OncoTargets and Therapy

17 May 2016

Number of times this article has been viewed

\section{Peipei $\mathrm{Xu}^{1, *}$ \\ Ruju Wang ${ }^{1,2, *}$ \\ Xiaohui Wang' \\ Jian Ouyang'}

'Department of Hematology, The Affiliated Drum Tower Hospital of Nanjing University Medical School, ${ }^{2}$ Medical School, Southeast University, Nanjing, People's Republic of China

*These authors contributed equally to this work
Correspondence: Peipei Xu; Jian Ouyang Department of Hematology, The Affiliated Drum Tower Hospital of Nanjing University Medical School, 321 Zhongshan Road, Nanjing 210008, People's Republic of China

Tel +86 I30 7259 0562;

$+862583105211$

Emailxu_peipei0618@I63.com; ouyangi21I@I63.com

\begin{abstract}
In the past few years, nanomaterial-based drug delivery systems have been applied to enhance the efficacy of therapeutics and to alleviate negative effects through the controlled delivery of targeting and releasing agents. However, few drug carriers can achieve high targeting efficacy, even when targeting modalities and surface markers are introduced. Immunological problems have also limited their wide applications. Biological drug delivery systems, such as erythrocytes, platelets, and albumin, have been extensively investigated because of their unique properties. In this review, erythrocytes, platelets, and albumin are described as efficient drug delivery systems. Their properties, applications, advantages, and limitations in disease treatment are explained. This review confirms that these systems can be used to facilitate a specific, biocompatible, and smart drug delivery.
\end{abstract}

Keywords: albumin, biological drug delivery systems, erythrocyte, platelet

\section{Introduction}

Drug delivery systems aim to improve the pharmacological properties of drugs and to achieve maximal therapeutic efficacy and minimal side effects by directing therapeutic cargos to target cells and tissues. ${ }^{1,2}$ Novel drug delivery systems have been developed because conventional and new drugs provide intrinsic disadvantages, such as poor stability, poor solubility, gastrointestinal reactions, and unwanted toxicity.

With remarkable advancements in nanotechnology, nanomaterials and nanostructures have been widely applied. Nanotechnology- and nanoscience-related studies have been extensively conducted over the past decades. Nanomaterial-based drug delivery systems have also been rapidly developed. ${ }^{3,4}$ Various nanomaterials, including nanoparticles, ${ }^{5}$ polymeric micelles, ${ }^{6}{ }^{6}$ lipsomes, ${ }^{7}$ and nanotubes ${ }^{8}$ have been utilized as drug carriers. Nanocarrier-delivered drugs provide several advantages over free drugs; for instance, nanocarriers can prolong circulation time, slow down metabolism, induce controlled drug release, and improve patient convenience and compliance. ${ }^{9}$ When used for chemotherapy, nanocarriers can enhance drug targeting to tumors because of their selective accumulation in tumors through enhanced permeability and retention (EPR) effects. EPR effects or passive targeting effects that often occur in solid tumors involve nanocarriers or macromolecules that preferentially accumulate in tumors rather than in normal tissues, because of leaky vasculature and poor lymphatic drainage in tumor cells and tissues. ${ }^{10}$ However, some hypoxic regions of solid tumors may fail to elicit EPR effects because of poor angiogenesis. ${ }^{11}$ Thus, active targeting strategies, including coupling with homing peptides, ${ }^{12}$ antibodies, ${ }^{13,14}$ and growth factors, ${ }^{15}$ have been employed to overcome the limitations of passive targeting. Although major advancements have been observed in this field, neither passive targeting nor active targeting can achieve 
specific nonrandom targeting that remains chance dependent. Human bodies are equipped with innate immune defenses, such as the reticuloendothelial system (RES), which rapidly recognizes and destroys foreign objects. As such, nanocarriers may be engulfed by macrophages of the mononuclear phagocyte system before these carriers reach target sites. ${ }^{16}$ As the immune system catches up in the game of hide and seek, biological drug carriers have been developed to bypass immune surveillance. ${ }^{16,17}$

Biological carriers, such as erythrocytes, platelets, and albumin, can bypass immune surveillance and provide several advantages, including long circulation time, good biodegradability, abundant surface ligands, and flexible morphology. Therefore, these carriers are optimum biological delivery systems and potential solutions to overcome the limitations of nanomaterial-based drug carriers. ${ }^{18,19}$ This review describes erythrocytes, platelets, and albumin as promising biological drug carriers and their applications in various diseases.

\section{Erythrocytes as drug delivery systems \\ Properties of erythrocytes}

Erythrocytes or red blood cells (RBCs) are enucleated biconcave disk-shaped cells in humans. RBCs are characterized by a diameter of $\sim 7 \mu \mathrm{m}$, a thickness of $\sim 2 \mu \mathrm{m}$, and a plasma membrane surface area of $\sim 160 \mu \mathrm{m}^{2}$. Erythrocytes are the most abundant blood components, and $1 \mu \mathrm{L}$ of human blood contains $\sim 4-5$ million RBCs. The half-life of human erythrocytes ranges from 100 days to 120 days. Various receptors, proteins, and functional groups on the membrane of erythrocytes provide binding sites for antibodies, specific ligands, and drug. ${ }^{20-22}$

The complex and unique membranes of RBCs are involved in the delivery of oxygen from lungs to different tissues. ${ }^{23}$ RBCs travel $\sim 250 \mathrm{~km}$ through the cardiovascular system containing capillary networks, which can be as narrow as one-third of a diameter of cells. ${ }^{24}$ The cytoskeleton, especially hexagonal actin-spectrin lattice underlying the plasmalemma, supports the membrane of RBCs, and the integrity of the cytoskeleton is based on a series of "horizontal" and "vertical" linkages between membrane protein complexes within the fluid phospholipid membrane bilayer. These structures ensure that the erythrocyte membrane simultaneously undergoes reversible changes between expanded and tight networks to maintain integrity and to avoid intravascular hemolysis. ${ }^{25}$ These damaged and aged RBCs are eliminated by tissue macrophages in the liver and spleen, and RBCs continuously circulate within the bloodstream but pass through the interstitium in splenic follicles and hepatic sinusoid. Thus, RBCs are suitable for intravascular delivery to treat blood, or endothelium-related diseases. ${ }^{1,26}$ Erythrocytes can be supplied as ideal drug delivery systems because of these important features; since the discovery of RBCs as potential carrier systems, numerous substances have been bound to these blood cells or used to encapsulate them for disease treatment. ${ }^{27-32}$

\section{Preparation approaches for RBCs as drug delivery systems \\ Encapsulation}

Several methods have been used to encapsulate cargos in RBCs. Osmosis-based methods developed in the 1970s have been considered standard methods, because erythrocytes can act as an osmometer. In a hypotonic solution, erythrocytes reversibly swell to $\sim 25 \%$ of their original volume, and pores ranging from $200 \AA$ to $500 \AA$ are temporarily formed in the membrane. Thus, substances can enter erythrocytes through these pores. In an isotonic solution, the shape of $\mathrm{RBCs}$ is regained, and their pores close; substances are then encapsulated within erythrocytes. ${ }^{33}$ This mechanism exhibits some variations, including hypotonic hemolysis, hypotonic pre-swelling, and hypotonic dilution. Considered as the most simple and rapid method of encapsulation, hypotonic dilution is the first process in which therapeutic moieties are loaded into erythrocytes. ${ }^{34-36}$

Another encapsulation method is electroporation or electro-insertion; in this process, erythrocytes are exposed to a strong external electrical field that causes irreversible changes in the RBC membrane. ${ }^{37,38}$ However, Kinosita and Tsong $^{39}$ suggested that desirable membrane permeability can be produced through transient electrolysis. Kitao et a ${ }^{40}$ pioneered the successful application of chemical perturbation in human and mouse RBCs in 1980. However, this method has been rarely employed because it induces irreversible destructive changes in the membrane. Alternative approaches, such as lipid fusion and endocytosis, have also been applied to encapsulate agents into erythrocytes. ${ }^{41,42}$

\section{Membrane coupling or binding}

Among conventional strategies, reversible or irreversible coupling of a therapeutic moiety to RBCs is the most extensively utilized strategy. Nonspecific chemical cross-linkers have been used to bind molecules to the surface of erythrocytes. ${ }^{43,44}$ However, implementation of these strategies has been impeded by specific cross-links, such as biotin-avidin pair, 
which allow biotinylated molecules, including nucleic acids, to couple to sulfhydryl groups, amino acids, and other specific groups expressed on the membrane of RBCs. Through these methods, various agents, such as viral antigens and immunoglobulins, have been coupled to the RBC surface. ${ }^{21,45}$ In addition to biotin-avidin, various chemical linkers have been utilized to attach different molecules, including small therapeutic molecules, such as daunorubicin, ${ }^{46}$ and large molecules, such as hyperbranched chemical linkers. ${ }^{47}$ Furthermore, single or combined molecules can conjugate with the surface of RBCs. In another approach, site-specific drug binding occurs as follows. ${ }^{48}$ Antibodies, peptides, or other affinity ligands are initially anchored to the surface proteins of erythrocytes; therapeutic agents bind to and conjugate with these ligands. ${ }^{48}$ Agents can also be attached to RBC surfaces through physical interactions; for instance, polystyrene particles can adhere to the surface of erythrocytes via nonspecific van der Waals and hydrogen bonding, which work through electrostatic and hydrophobic forces, respectively, between particles and erythrocytes. ${ }^{49}$

Figure 1 illustrates the two main preparation strategies for RBCs as a drug delivery system.

\section{Application of erythrocytes as a drug delivery system}

Erythrocytes encapsulate and protect L-asparaginase (L-ASNase) from degradation; the encapsulated L-ASNase exhibits a longer half-life and lower incidence and severity of side effects, such as allergic reactions than native L-ASNase. Encapsulated L-ASNase is a promising product with a good safety profile to treat children and adults with refractory or relapsing acute lymphoblastic leukemia. ${ }^{50,51}$ For instance, the toxic effect of erythrocyte-delivered etoposide on

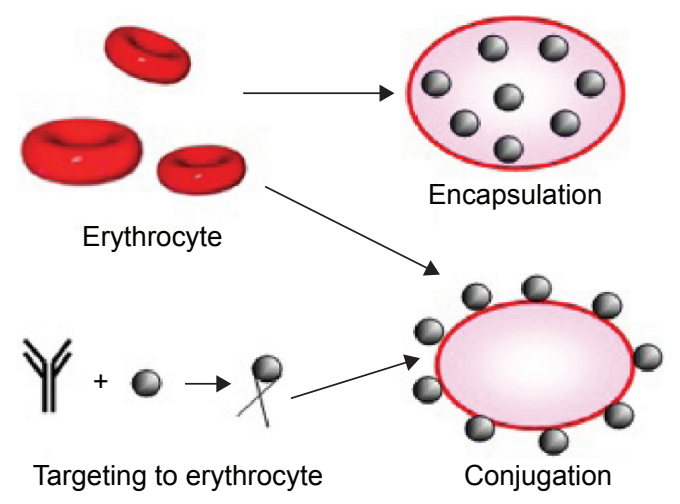

Figure I Two main strategies of preparing erythrocytes as drug delivery systems. Notes: In the method of encapsulation, therapeutic agents are inserted within erythrocytes, while in the method of conjugation, they are attached to the erythrocyte membrane. macrophages is greater than that of free chemotherapeutic drugs; this phenomenon indicates the usefulness of RBCs in the delivery of this cytotoxic agent to target macrophages. ${ }^{52}$ Mitochondrial neurogastrointestinal encephalomyopathy (MNGIE), an autosomal recessive disease, is caused by mutations in the nuclear gene ECGF1 coding for thymidine phosphorylase (TP); thus, high deoxythymidine and deoxyuridine levels accumulate in the body and induce gastrointestinal motility disorders, such as stomachache, progressive external ophthalmoplegia, hearing loss, and peripheral sensorimotor polyneuropathy. Moran et $\mathrm{al}^{53}$ found that deoxythymidine and deoxyuridine levels decrease significantly, and symptoms have been ameliorated in patients with MNGIE when TP is carried by RBCs. Levene et al concluded that serious toxicities likely preclude a clinical trial of TP carried by RBCs in patients with MNGIE. ${ }^{54}$ Harisa et al ${ }^{32}$ demonstrated that human erythrocytes can be successfully loaded with pravastatin, and relatively high drug loading and encapsulation efficiency can be obtained. Moreover, no significant loading parameters and morphological changes in erythrocytes have been observed in entrapping pravastatin; this finding indicates that erythrocytes are potential carriers for pravastatin. Methotrexate (MTX), an antimetabolite and antifolate agent used in solid tumors and hematological diseases, can be encapsulated by erythrocytes, and the average survival time of rat hepatoma cells is enhanced with MTXloaded erythrocyte treatment compared with that of cells treated with native MTX. ${ }^{55}$ Biagiotti et $\mathrm{al}^{56}$ confirmed that immunosuppressants can be encapsulated into erythrocytes in the presence of corresponding target proteins, and RBCs can serve as a promising delivery system for immunosuppressive agents. ${ }^{56}$ The use of RBCs as a drug delivery system for chemotherapeutic agents, especially in vitro and in vivo use of antineoplastic agents, has been widely investigated. Other therapeutic drugs delivered by RBCs include gentamicin for bacterial infection, ${ }^{57} \delta$-aminolevulinate dehydratase for lead poisoning, ${ }^{58} \beta$-glucocerebrosidase for enzyme replacement therapy in Gaucher's disease ${ }^{59}$ adenosine deaminase for adenosine deaminase deficiencies, ${ }^{60}$ enalaprilat for hypertension management and congestive heart failure, ${ }^{61}$ and heparin for thromboembolism ${ }^{62}$ and carrier for thrombolytic agents. ${ }^{63}$ Table 1 provides some examples of therapeutic moieties loaded by erythrocytes. ${ }^{28,46,64-75}$

\section{Advantages}

Erythrocytes applied as drug delivery systems have been extensively investigated because of several factors. For instance, erythrocyte sources are abundant, and the structure 
Table I Examples of therapeutic moieties loaded by erythrocytes

\begin{tabular}{|c|c|c|c|c|}
\hline Therapeutic moieties & Application & Approaches & Study types & References \\
\hline Dexamethasone & Crohn's disease; ulcerative colitis & Encapsulation & Both in vitro and in vivo & $64-66$ \\
\hline Dexamethasone & Chronic obstructive pulmonary disease & Encapsulation & In vivo & 67 \\
\hline Dexamethasone & Cystic fibrosis & Encapsulation & In vivo & 68 \\
\hline Dexamethasone & Ataxiateleangiectasia & Encapsulation & In vivo & 69 \\
\hline Daunorubicin & Acute leukemia & Binding & Both in vitro and in vivo & 46 \\
\hline Doxorubicin & Lymphoma & Binding & In vivo & 70 \\
\hline 5-Fluorouracil & Malignant ascites & Encapsulation & In vivo & 28 \\
\hline Phenylalanine ammonia lyase & Phenylketonuria & Encapsulation & In vivo & 71 \\
\hline IFN- $\alpha$ and Ribavirin & Hepatitis C & Encapsulation & In vitro & 72 \\
\hline Glutamine synthetase & Hyperammonemia & Encapsulation & In vivo & 73 \\
\hline Factor IX & Hemophilia B & Encapsulation & In vitro & 74 \\
\hline Inositol hexaphosphate & Sickle cell disease & Encapsulation & In vitro & 75 \\
\hline
\end{tabular}

Abbreviation: IFN- $\alpha$, interferon- $\alpha$.

and properties of erythrocytes are well understood. RBCs also possess good biocompatibility and biodegradability without inducing immunological reactions and producing toxic by-products. The membrane properties of RBCs permit relatively high drug loading and slow molecular release. Furthermore, their circulation time in the bloodstream is prolonged. RBCs are phagocytosed by macrophages in the liver and spleen. Thus, RBCs introduce cargos into the RES of cells, thus they can be beneficial for the treatment of macrophage-related hepatic diseases ${ }^{22,23,48,76}$ (Figure 2).

\section{Drawbacks}

Similar to other drug delivery systems, carrier erythrocytes are limited by various factors. For example, encapsulation may cause an osmotic stress-induced damage to the RBC membrane. Coupling therapeutic moieties to RBCs can lead to the loss of the mechanical stability and plasticity of erythrocytes. These morphological and physiological

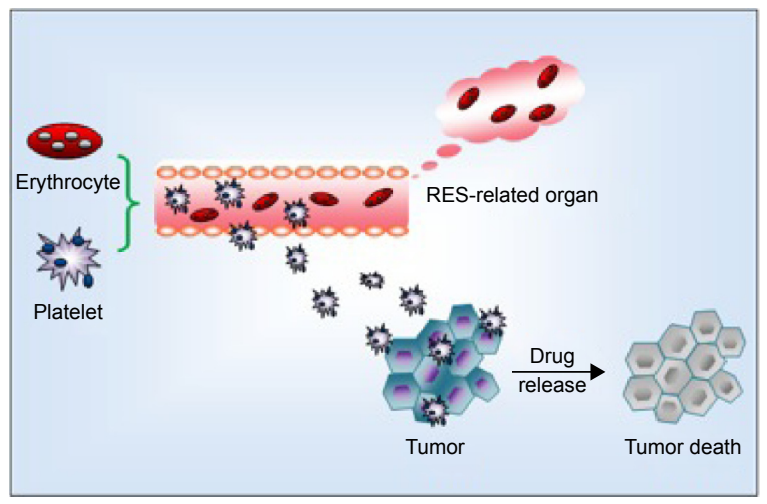

Figure 2 Illustration of the mechanism of erythrocytes and platelets as drug delivery systems.

Note: Erythrocytes are targeting to RES-related organs and platelets are targeting to tumor.

Abbreviation: RES, reticuloendothelial system. changes in erythrocytes may trigger an unwanted removal of RBCs by RES; as a consequence, their circulation time in the bloodstream is decreased. Molecules encapsulated in or coupled with RBCs may induce erythrocyte leakage and thus elicit toxic effects. Furthermore, preparation strategies for erythrocyte carriers have yet to be standardized; these carriers are also more varied than synthetic carrier systems. The storage of erythrocyte carriers and risk of blood contamination before, during, and after drug loading should also be highly considered. ${ }^{27,77}$

\section{Advancements}

Drug selection and RBC-drug relationship have been improved because of various limitations. Prodrugs, such as corticosteroid prodrugs for prolonged circulation and nucleoside/nucleotide prodrugs for macrophage targeting, can improve the performance of RBC-based drug carriers. If an encapsulated or coupled molecule is inactive, erythrocytes can transform and release prodrugs in their active forms. This strategy can reduce off-target effects and prolong the half-life of drugs. Kwon et $\mathrm{al}^{78}$ introduced a new strategy of protein loading by using membrane-translocating low molecular weight protamine, which causes negligible changes in RBC membrane. Other novel engineered substances, such as nanoparticles and polymeric multilayered microcapsules, combined with RBCs are also currently used. ${ }^{22,79,80}$

\section{Platelets as drug delivery systems Properties of platelets}

Platelets, with an average diameter of $2-3 \mu \mathrm{m}$, are discoid fragments from megakaryocytes of the bone marrow and the smallest components of the blood. Approximately $10^{8}$ cells can be produced by megakaryocytes daily. Thus, $2 \times 10^{11}$ to $5 \times 10^{11}$ platelets can be generated; when demands are high, 
this number increases tenfold. The platelet concentration in the circulating blood is $\sim 150 \times 10^{9} / \mathrm{L}$ to $350 \times 10^{9} / \mathrm{L}$ with an average life span of 7-10 days; after this period, platelets are removed by reticuloendothelial cells from the liver and spleen. ${ }^{81,82}$

Platelets participate in several physiological and pathological processes, including hemostasis, wound healing, thrombosis, inflammation, and atherosclerosis. ${ }^{83}$ In hemostasis, damaged small blood vessels and capillaries promote vasoconstriction to prevent blood loss. In addition, platelets adhere to the site of injury by interacting with von Willebrand factor and collagen; some mediators work with various potent functional molecules, including adenosine diphosphate and thromboxane A2. Thus, platelets act as a storage "depot" and trafficking "vehicle" in blood vessels. ${ }^{84}$

Pathological conditions occur because of nonspecific activity of platelets, stimulation of agonist secretion, and platelet aggregation in atheromatous plaques; these conditions decrease the number of platelets in circulation and cause ischemia. ${ }^{85}$ The mechanism by which platelets engulf small molecules is different from conventional phagocytosis, in which an engulfed substance is metabolized. Compared with other carriers, encapsulated substances in the platelet remain intact, and are thus referred to as covercytes. ${ }^{86}$

The relationship between platelets and malignancy was first discovered in 1865 by Armand Trousseau, a French clinician who reported that migratory thrombophlebitis can be an indicator of occult malignancies, including pancreatic carcinoma. Named after this clinician, Trousseau syndrome is characterized by a hypercoagulable state with advanced malignancy. Seven years later, Riess ${ }^{87}$ described a "massive increase of platelets" in patients with carcinoma. In $1878,{ }^{88}$ Billroth found that platelets not only participate in tumor growth but also contribute to metastatic spread. A century later, researchers found that thrombocytopenia alleviates metastasis in mice. Tumor cells induce platelet aggregation, and active platelets protect or cloak circulatory tumor cells from physical factors such as shear stress within the vasculature, and help them escape from immune surveillance. Subsequently, platelets cause tumor cells to transfer to secondary sites on the vascular wall; platelets also secrete proteases and cytokines that promote the formation of new blood vessels, which are necessary for tumor-associated angiogenesis and growth. Platelet count is increased by tumor cells. Therefore, the correlation between platelets and tumors can be described as a vicious cycle: tumor increases platelet count; the increased platelet count promotes tumor growth and metastasis; as a consequence, platelet count is further increased (Figure 3). ${ }^{89,90}$ The phenomenon by which tumor cells cause platelet aggregation was first discovered in 1968 and referred to as tumor-induced platelet aggregation (TCIPA). ${ }^{91}$ TCIPA is considered the fundamental to utilize platelets as drug delivery systems for malignant tumor treatment. The mechanism of TCIPA is illustrated in Figure 2.

\section{Application of platelets as a drug delivery system}

Sarkar et $\mathrm{al}^{92}$ showed that platelets can take up drugs at a relatively high drug loading concentration, and the efficiency of inducing cytotoxicity is significantly increased when platelets are used as drug delivery carriers in vitro and in vivo. They concluded that platelets can be potential drug carriers when a low drug concentration is used in a targeted mode. Antithrombotic agents are commonly utilized to manage patients at a high risk of thrombosis and hemorrhage. Platelets have also been effectively exploited as carriers for antithrombotic agents, especially for arterial clots. ${ }^{93,94}$ Davies et $\mathrm{al}^{95}$ constructed a strategy to deliver europium luminescent complex and gold nanoparticles (AuNPs) into human platelets by using a low-pH insertion peptide. The radiolabeling of platelets to assess survival and recovery has been developed for more than 4 decades. However, this technique has been banned for use in clinical studies. By contrast, nonradioactive labeling of platelets has been used frequently in clinical studies, including evaluation of the survival of transfused platelets and analysis of the pathogenesis of stroke. ${ }^{96}$ In our study,

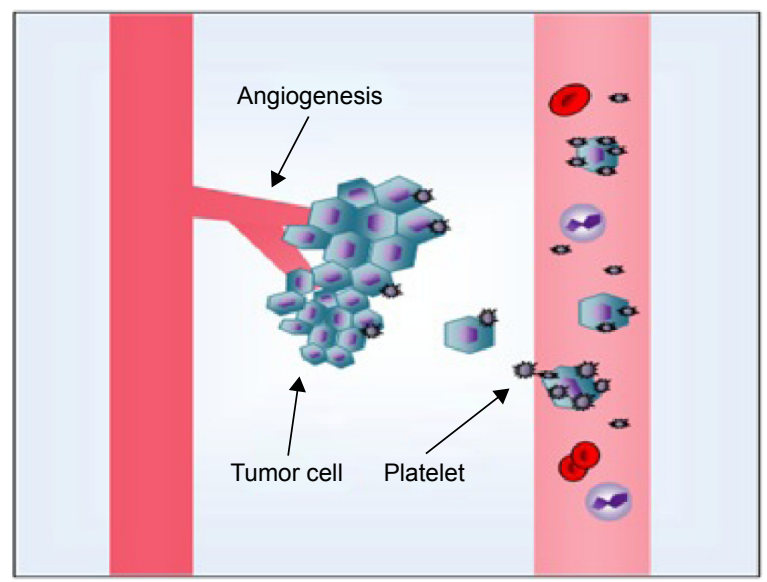

Figure 3 The relationship between platelets and malignancy.

Notes: Tumor cells induce platelet aggregation, and then active platelets protect or cloak circulatory tumor cells from physical factors, as well as help them escape from immune surveillance. Subsequently, platelets facilitate tumor cells to transfer to the secondary sites on the vascular wall and secrete proteases and cytokines that promote the formation of new blood vessels. On the other hand, tumor cells increase the platelet count. 
Table 2 Examples of therapeutic moieties loaded by platelets

\begin{tabular}{|c|c|c|c|}
\hline Therapeutic moieties & Application & Study types & References \\
\hline Factor VIII & Hemophilia A & In vivo & 101 \\
\hline Factor IX & Hemophilia B & In vivo & 84 \\
\hline Doxorubicin & Lung adenocarcinoma; ehrlich ascites carcinoma & Both in vitro and in vivo & 92 \\
\hline Ferucarbotran superparamagnetic nanoparticles & Transfusion medicine and cardiovascular medicine & In vitro & 96 \\
\hline
\end{tabular}

doxorubicin is loaded into platelets, and high drug loading and encapsulation efficacy are detected. Doxorubicin is also released by a pH-based trigger. Inhibition and apoptosis rates are higher in the group that received drug-loaded platelets than in the untreated group.

Nanoparticles mimicking platelets have been proposed for drug and gene delivery to treat various diseases. Kona et a ${ }^{97}$ formulated drug-loaded poly(lactic-co-glycolic acid) nanoparticles and conjugated the external fraction of platelet glycoprotein Ib (GPIb) via carbodiimide chemistry. They confirmed that the GPIb-conjugated poly(lactic-co-glycolic acid) nanoparticles can act as a controlled and targeted drug carrier under flow conditions at vascular injury sites. ${ }^{97}$ Lin et al obtained similar results. ${ }^{98}$ Modery-Pawlowski et al ${ }^{99}$ found that platelet mimetics is a promising method in the treatment of metastasized tumor. Researchers prepared polymeric nanoparticles enclosed in the plasma membrane of human platelet; they verified that platelet membrane-cloaked nanoparticles exhibit less uptake by macrophage-like cells than uncoated nanoparticles do, and particle-induced complement activation is reduced. Furthermore, therapeutic efficacy is enhanced when vancomycin and docetaxel are applied to an experimental mouse model of systemic bacterial infection and a rat model of coronary restenosis via platelet-mimetic nanoparticles. ${ }^{100}$

Other applications of platelets as drug carriers are summarized in Table 2.92,101

\section{Advantages}

Biocompatibility, which is defined as "expression of the benignity of the relation between a material and its biological environment", is considered a fundamental property of a carrier. The biocompatibility of platelets is superior to that of other drug delivery systems because platelets are components of the human body. In theory, $\sim 500$ molecules can be loaded on $1 \times 10^{-15} / \mathrm{L}$ nanoparticles; in reality, the amount is much lower. The loading efficacy of platelets is relatively high, with an average of 50,000-70,000 molecules encapsulated by the same number of platelets. ${ }^{86,102}$ Another property of platelets is that targets are well defined: these targets are mostly sites with high density of proliferating cells or injured sites. Thus, side effects caused by nonspecific targeting are decreased because of the inherent targeting capacity of platelets. Furthermore, platelet-encapsulated drugs are protected from physical stress and immunological reactions; as such, the systemic clearance of the encapsulated drugs is similar to that of single platelet, and circulation time is prolonged. Controlled drug delivery is quite important, and platelets can facilitate controlled drug release by artificially exposing drugs to agonists. According to Sarkar et al, lethal effects are exacerbated by enhanced apoptosis when cytotoxic drugs are carried by platelets; this phenomenon indicates the synergetic effect of platelets and drugs. As such, low amounts of drugs can be used to achieve the same treatment efficacy, and few side effects are induced. Another advantage of platelets over other carriers is that the method can be individualized because a patient's own platelets are the ones used to deliver drugs. ${ }^{92}$ The method is also appropriate for patients who need transfusion.

\section{Drawbacks}

The main drawbacks of platelets are similar to those of RBCs. These limitations include proper storage and contamination. In genetic therapy, whether the platelet-specific expression of transgenes affects the expression of endogenous proteins remains unknown. Furthermore, whether the usage of platelets as drug carriers causes thrombogenesis should be further investigated.

\section{Albumin as a drug delivery system Properties of albumin and its preparation}

Albumin, which is the most abundant plasma protein, acts as a molecular taxi for various small insoluble substances, such as hormones, nutrients, and toxins. The albumin concentration in an adult ranges from $35 \mathrm{~g} / \mathrm{L}$ to $50 \mathrm{~g} / \mathrm{L} .{ }^{103}$ Albumin, with an average half-life of 19 days, is synthesized in the liver and its molecular weight is $66.5 \mathrm{kDa} .{ }^{104}$

Babson and Winnick ${ }^{105}$ demonstrated for the first time that tumor cells can trap plasma proteins and utilize their degradation products for proliferation. Since then, other researchers have revealed the ability of albumin to accumulate in malignant tissues via the EPR effect. The leakiness of the defective blood vessels of tumor tissues enables macromolecules to pass the endothelial barrier, but normal 
tissues are permeable only to small molecules. ${ }^{71,104}$ As such, the proper size of macromolecules that can extravasate into tumor tissues but not into healthy tissues ranges from $2 \mathrm{~nm}$ to $10 \mathrm{~nm}$, and the diameter of serum albumin is $7.2 \mathrm{~nm}$. In addition, Stehle et al proposed that albumin is a major nutrient and energy source for tumor cell growth. ${ }^{106}$ In 2003, Wunder et a ${ }^{107}$ showed that albumin accumulates in the arthritic paws of mice. Therefore, albumin is a potential carrier that can be used to deliver drugs to the inflamed joints of patients with rheumatoid arthritis. ${ }^{104}$

Albumin as a drug delivery system is prepared via two primary ways: 1) encapsulation of drugs into albumin nanoparticles, which are dependent on physical interaction between drugs and albumin, and 2) coupling of drugs to endogenous or exogenous albumin and conjugation with bioactive proteins.

\section{Application of albumin as a drug delivery system}

The MTX bound to human serum albumin (MTX-HSA) is the first albumin conjugate evaluated in Phase I/II clinical studies. In the assessment of antitumor activity in preclinical in vivo models, complete remission/cure is achieved in softtissue sarcoma SXF 1301 by using MTX-HSA; free MTX lasts for a short period. In the prostate cancer model PRXF PC3M, MTX-HSA induces a greater growth inhibition than native MTX dose. These results demonstrate that antitumor effectiveness can be enhanced when MTX is conjugated with albumin. ${ }^{108}$ MTX-HSA can be combined with cisplatin, and the toxicity of the antitumor activity against urothelial carcinomas is tolerable. ${ }^{109}$ One of the successful applications of albumin as a drug delivery system is demonstrated by the albumin-paclitaxel (PAC) nanoparticle Abraxane, which was first approved in 2005 by the US Food and Drug Administration to treat metastatic breast cancer; this drug was also approved in 41 countries in the same year. Teneriello et al ${ }^{110}$ verified that nanoparticle albumin-bound PAC is active in patients with recurrent ovarian, peritoneal, or fallopian tube cancer, and toxicity is manageable, as revealed by Phase II evaluation. HSA has been proposed as a carrier for renal targeting drugs, but glomeruli are not entirely permeable to HSA because of the molecular weight of albumin. Therefore, Yuan et al ${ }^{111}$ used three peptide fragments (PFs), namely PF-A1-123, PF-A124-298, and PF-A299-585, obtained through the cyanogen bromide degradation of HSA to carry agents and selectively accumulate in kidneys; they verified that PFs can be applied as potential drug delivery systems for renal targeting, and PF-A299-585 may be an optimal carrier. Cationic bovine serum albumin as a gene delivery system has also been demonstrated as a promising strategy to treat lung and metastatic cancers. ${ }^{112}$ Asmatulu et al ${ }^{113}$ fabricated albumin-based drug-carrying microcomposite spheres loaded with cyclophosphamide and 5-fluorouracil; they subsequently investigated the potential of these spheres for breast cancer treatment in vitro by using fibroblast cells (3T3) and breast cancer cells (MDA-486). Nude mice have also been used for in vivo evaluation. The results showed that chemotherapeutic efficacy is enhanced when the mice are exposed to other drug delivery systems possibly because of the albumin-induced increase in uptake of microspheres by malignant cells. Peralta et $\mathrm{al}^{114}$ revealed that gold nanorods combined with the chemotherapeutic drug PAC are successfully encapsulated into HSA nanoparticles (HSAPs) to form PAC-AuNP-HSAPs. They verified that PAC-AuNPHSAPs are more efficient than free PAC in inhibiting the proliferation of 4T1 mouse breast cancer cells and inducing the apoptosis of these cancer cells. Zhang et al ${ }^{115}$ demonstrated that cytotoxicity is enhanced by two to five times in tumor cells when ruthenium-based anticancer complexes [RuCl5(ind) $]^{2-}$ bind to HSA; no side effects are detected in normal cells in vitro. Compared with the use of single drugs, the use of the HSA-[RuCl5(ind) $]^{2-}$ complex promotes tumor cell apoptosis. Albumin drug nanoparticles combined with phospholipid perifosine or the antibodies trastuzumab (Herceptin) and bevacizumab (Avastin) can effectively treat patients with head, neck, or breast cancer. ${ }^{116}$ Thus, albumin is a potential drug delivery system. ${ }^{117}$ Other uses of albumin as a drug carrier are listed in Table 3. ${ }^{112,118-127}$

\section{Advantages}

Albumin is an optimal candidate for drug delivery because of several advantages, including abundant sources, nontoxicity, biodegradability, nonimmunogenicity, and preferential uptake in tumor and inflamed tissues. Albumin is also stable at various temperatures (it can be heated at $60^{\circ} \mathrm{C}$ for up to 10 hours without deleterious effects), $\mathrm{pH}$ (it is stable at $\mathrm{pH} 4-9$ ), and in various organic solvents. ${ }^{117}$ Therefore, albumin can enhance the solubility of poorly water-soluble molecules, prolong the circulation time of drugs, and minimize side effects by improving targeting. Furthermore, albumin provides a depot for various drug binding sites and high binding capacity for different drugs. ${ }^{117,128}$

\section{Drawbacks}

In preparing albumin-drug conjugates with or without additional targeting ligands, the structure of albumin may 
Table 3 Examples of therapeutic moieties loaded by albumin

\begin{tabular}{|c|c|c|c|c|}
\hline Therapeutic moieties & Application & Approaches & Study types & References \\
\hline Deferasirox & Neurodegenerative disorders & Conjugation & In vitro & 118 \\
\hline Noscapine & Breast cancer & Coacervation & In vitro & 119 \\
\hline Tacrolimus & Rheumatoid arthritis tissues & Conjugation & Both in vitro and in vivo & 120 \\
\hline Azidothymidine & AIDS & Conjugation & In vitro & 121,122 \\
\hline Exendin-4 & Type 2 antidiabetic & Conjugation & In vivo & 123 \\
\hline Aspirin & Intra-articular therapy & Coacervation & In vitro & 124 \\
\hline Obidoxime & Poisoning by organophosphorus compounds & Conjugation & In vitro & 125,126 \\
\hline siRNA & Lung metastatic cancer & Encapsulation & Both in vitro and in vivo & 112 \\
\hline Doxorubicin & $\begin{array}{l}\text { Breast carcinoma; ovarian carcinoma; small } \\
\text { cell lung cancer; orthotopic pancreas carcinoma }\end{array}$ & Conjugation & Both in vitro and in vivo & 127 \\
\hline
\end{tabular}

Abbreviation: siRNA, small interfering RNA.

be disrupted; as a result, these conjugates may accumulate in nontarget tissues and may be eradicated by the mononuclear phagocyte sysetm. ${ }^{128}$ Furthermore, the performances of nanomaterials and albumin in vitro differ from the interaction of albumin and nanomaterials in vivo; undesirable results, such as the risk of capillary blockage because of increased size and potential toxicity caused by conformational changes in albumin, may also be obtained. ${ }^{9}$

\section{Others}

In addition to erythrocytes, platelets, and albumin as drug delivery systems, apoferritin has been used to encapsulate cisplatin and carboplatin. ${ }^{129}$ Ivy nanoparticles also exhibit great potential for the delivery of chemotherapeutic drugs in cancer therapy. ${ }^{130}$ Wang et al ${ }^{131}$ suggested that tea nanoparticles can be useful to prevent multidrug resistance in tumor cells and to enhance the chemotherapeutic efficacy in tumor treatment. Yi et al ${ }^{132}$ also confirmed that tea nanoparticles can function as a multifunctional nanocarrier for cancer therapy. Fungal nanoparticles have also been proposed as nanocarriers for cancer treatment. ${ }^{133}$ Low-density lipoprotein, ${ }^{134}$ nonpathogenic bacteria, ${ }^{135,136}$ and dendritic and eukaryotic cells ${ }^{137}$ have also been used to deliver drugs, enzymes, and genes to specific sites.

\section{Conclusion}

Biological agents have been investigated as drug delivery systems in various research and clinical applications, especially for antineoplastic agent delivery, because of their excellent properties. The advantages of biological delivery carriers have been verified by numerous studies. Nevertheless, further research should be conducted to clarify the following questions: 1) Can thrombus be formed through the reduplicative application of platelets as drug carriers? 2) Can carrier erythrocytes deliver oxygen from lungs to different tissues? 3) Can carrier platelets still participate in physiological and pathological processes; if so, what is their role in human bodies?

\section{Acknowledgments}

We would like to thank all the volunteers who took part in this study. This work was supported by the National Natural Science Foundation of China (81400162, 81570174), the Natural Science Foundation of Jiangsu Province (BK20140100), the Technique Development Foundation of Nanjing (Outstanding Youth Foundation, JQX15004), the Innovation Program of Jiangsu Province (SJZZ15_0029), and the Medical Science and Technology Development Program of Nanjing (Ykk14069, 201402066).

\section{Disclosure}

The authors report no conflicts of interest in this work and have received no payment in preparation of this manuscript.

\section{References}

1. Yoo JW, Irvine DJ, Discher DE, Mitragotri S. Bio-inspired, bioengineered and biomimetic drug delivery carriers. Nat Rev Drug Discov. 2011; 10(7):521-535.

2. Lai JY. Biodegradable in situ gelling delivery systems containing pilocarpine as new antiglaucoma formulations: effect of a mercaptoacetic acid/N-isopropylacrylamide molar ratio. Drug Des Devel Ther. 2013; 7:1273-1285.

3. Markman JL, Rekechenetskiy A, Holler E, Ljubimova JY. Nanomedicine therapeutic approaches to overcome cancer drug resistance. Adv Drug Deliv Rev. 2013;65(13-14):1866-1879.

4. Hou L, Yang XM, Ren JX, et al. A novel redox-sensitive system based on single-walled carbon nanotubes for chemo-photothermal therapy and magnetic resonance imaging. Int J Nanomedicine. 2016;11:607-624.

5. Fukumori $Y$, Ichikawa $H$. Nanoparticles for cancer therapy and diagnosis. Adv Powder Technol. 2006;17(1):1-28.

6. Cheng Q, Du LL, Meng LW, et al. The promising nanocarrier for doxorubicin and siRNA co-delivery by PDMAEMA-based amphiphilic nanomicelles. ACS Appl Mater Interfaces. 2016;8(7):4347-4356.

7. Shigehiro T, Zhai W, Vaidyanath A, et al. Evaluation of glycosylated docetaxel-encapsulated liposomes prepared by remote loading under solubility gradient. J Microencapsul. 2016;33(2):172-182.

8. Wu PP, Li S, Zhang HJ. Design real-time reversal of tumor multidrug resistance cleverly with shortened carbon nanotubes. Drug Des Devel Ther. 2014;8:2431-2438. 
9. Peng $\mathrm{Q}, \mathrm{Mu} \mathrm{H}$. The potential of protein-nanomaterial interaction for advanced drug delivery. J Control Release. 2016;225:121-132.

10. Kim J, Kim PH, Kim SW, Yun CO. Enhancing the therapeutic efficacy of adenovirus in combination with biomaterials. Biomaterials. 2012;33(6): $1838-1850$.

11. Fang J, Nakamura H, Maeda H. The EPR effect: unique features of tumor blood vessels for drug delivery, factors involved, and limitations and augmentation of the effect. Adv Drug Deliv Rev. 2011;63(3): 136-151.

12. Yao XL, Yoshioka Y, Morishige T, et al. Tumor vascular targeted delivery of polymer-conjugated adenovirus vector for cancer gene therapy. Mol Ther. 2011;19(9):1619-1625.

13. Bachtarzi H, Stevenson M, Ubr V, Seymour LW, Fisher KD. E-selectin is a viable route of infection for polymer-coated adenovirus retargeting in TNF- $\alpha$-activated human umbilical vein endothelial cells. $J$ Drug Target. 2011;19(8):690-700.

14. Willemsen RA, Pechar M, Carlisle RC, et al. Multi-component polymeric system for tumour cell-specific gene delivery using a universal bungarotoxin linker. Pharm Res. 2010;27(11):2274-2282.

15. Park JW, Mok H, Park TG. Epidermal growth factor (EGF) receptor targeted delivery of PEGylated adenovirus. Biochem Biophys Res Commun. 2008;366(3):769-774.

16. Su Y, Xie Z, Kim GB, Dong C, Yang J. Design strategies and applications of circulating cell-mediated drug delivery systems. ACS Biomater Sci Eng. 2015;1(4):201-217.

17. Fang RH, Hu CM, Zhang L. Nanoparticles disguised as red blood cells to evade the immune system. Expert Opin Biol Ther. 2012;12(4): 385-389.

18. Hamidi M, Zarrin A, Foroozesh M, Mohammadi-Samani S. Applications of carrier erythrocytes in delivery of biopharmaceuticals. J Control Release. 2007;118(2):145-160.

19. Zhang F, Xu CL, Liu CM. Drug delivery strategies to enhance the permeability of the blood-brain barrier for treatment of glioma. Drug Des Devel Ther. 2015;9:2089-2100.

20. Allen TM. Ligand-targeted therapeutics in anticancer therapy. Nat Rev Cancer. 2002;2(10):750-763.

21. Muzykantov VR, Taylor RP. Attachment of biotinylated antibody to red blood cells: antigen-binding capacity of immunoerythrocytes and their susceptibility to lysis by complement. Anal Biochem. 1994;223(1) $142-148$.

22. Bhateria M, Rachumallu R, Singh R, Bhatta RS. Erythrocytes based synthetic delivery systems: transition from conventional to novel engineering strategies. Expert Opin on Drug Deliv. 2014;11(8):1219-1236.

23. Muzykantov VR. Drug delivery by red blood cells: vascular carriers designed by mother nature. Expert Opin Drug Deliv. 2010;7(4): 403-427.

24. Sosa JM, Nielsen ND, Vignes SM, Chen TG, Shevkoplyas SS. The relationship between red blood cell deformability metrics and perfusion of an artificial microvascular network. Clin Hemorheol Microcirc. 2013;57(3):275-289.

25. Villa CH, Pan DC, Zaltsev S, Cines DB, Siegel DL, Muzykantoy VR. Delivery of drugs bound to erythrocytes: new avenues for an old intravascular carrier. Ther Deliv. 2015;6(7):795-826.

26. Oldenborg PA, Zheleznyak A, Fang YF, Lagenaur CF, Gresham HD, Lindberg FP. Role of CD47 as a marker of self on red blood cells. Science. 2000;288(5473):2051-2054.

27. Millan CG, Marinero ML, Castaneda AZ, Lanao JM. Drug, enzyme and peptide delivery using erythrocytes as carriers. $J$ Control Release. 2004;95(1):27-49.

28. Wang GP, Guan YS, Jin XR, et al. Development of novel 5-fluorouracil carrier erythrocyte with pharmacokinetics and potent antitumor activity in mice bearing malignant ascites. $J$ Gastroenterol Hepatol. 2010;25(5):985-990.

29. Alanazi FK, Haria Gel-D, Maqboul A, Abdel-Hamid M, Neau SH, Alsarra IA. Biochemically altered human erythrocytes as a carrier for targeted delivery of primaquine: an in vitro study. Arch Pharm Res. 2011;34(4):563-571.
30. Staedtke V, Brahler M, Muller A, et al. In vitro inhibition of fungal activity by macrophage-mediated sequestration and release of encapsulated amphotericin B nanosuspension in red blood cells. Small. 2010;6(1):96-103.

31. Shavi GV, Doijad RC, Deshpande PB, et al. Erythrocytes as carrier for prednisolone: in vitro and in vivo evaluation. Pak J Pharm Sci. 2010;23(2):194-200.

32. Harisa Gel D, Ibrahim MF, Alanazi FK. Characterization of human erythrocytes as potential carrier for pravastatin: an in vitro study. Int J Med Sci. 2011;8(3):222-230.

33. Kwant W, Seeman P. The erythrocyte ghost is a perfect osmometer. J Gen Physiol. 1970;55(2):208-219.

34. Ihler GM, Glew RH, Schnure FW. Enzyme loading of erythrocytes. Proc Natl Acad Sci U S A. 1973;70(9):2663-2666.

35. DeLoach J, Harris R, Ihler G. An erythrocyte encapsulator dialyzer used in preparing large quantities of erythrocyte ghosts and encapsulation of a pesticide in erythrocyte ghosts. Anal Biochem. 1980;102(1): 220-227.

36. Tajerzadeh H, Hamidi M. Evaluation of hypotonic preswelling method for encapsulation of enalaprilat in intact human erythrocytes. Drug Dev Ind Pharm. 2000;26(12):1247-1257.

37. Lizano C, Perez MT, Pinilla M. Mouse erythrocytes as carriers for coencapsulated alcohol and aldehyde dehydrogenase obtained by electroporation in vivo survival rate in circulation, organ distribution and ethanol degradation. Life Sci. 2001;68(17):2001-2016.

38. Dong Q, Jin W. Monitoring diclofenac sodium in single human erythrocytes introduced by electroporation using capillary zone electrophoresis with electrochemical detection. Electrophoresis. 2001; 22(13):2786-2792.

39. Kinosita K, Tsong T. Hemolysis of human erythrocytes by transient electric field. Proc Natl Acad Sci U S A. 1977;74(5):1923-1927.

40. Kitao T, Hattori K, Takeshita M. Agglutination of leukemia cells and daunomycin entrapped erythrocytes with lectin in vitro and in vivo. Experientia. 1978;34(1):94-95.

41. Nicolau C, Gersonde K. Incorporation of inositol hexaphosphate into intact red blood cells. Naturwissenschaften. 1979;66(11):563-566.

42. Schriei S, Bensch K, Johnson M, Junga I. Energized endocytosis in human erythrocyte ghosts. J Clin Invest. 1975;56(1):8-22.

43. Page Faulk W, Houba V. Immunological reactions with chromic chloride-treated erythrocytes. J Immunol Methods. 1973;3(1): 87-98.

44. Boyden SV. The adsorption of proteins on erythrocytes treated with tannic acid and subsequent hemagglutination by antiprotein sera. $J$ Exp Med. 1951;93(2):107-120.

45. Corinti S, Chiarantini L, Dominici S, Laguardia ME, Magnani M, Girolomoni G. Erythrocytes deliver Tat to interferon-gamma-treated human dendritic cells for efficient initiation of specific type 1 immune responses in vitro. J Leukoc Biol. 2002;71(4):652-658.

46. Skorokhod OA, Garmaeva TT, Vitvitsky VM, et al. Pharmacokinetics of erythrocyte-bound daunorubicin in patients with acute leukemia. Med Sci Monit. 2004;10(4):PI55-PI64.

47. Rossi NA, Constantinescu I, Kainthan RK, Brooks DE, Scott MD, Kizhakkedathu JN. Red blood cell membrane grafting of multi-functional hyperbranched polyglycerols. Biomaterials. 2010;31(14):4167-4178.

48. Hu CM, Fang RH, Zhang L. Erythrocyte-inspired delivery systems. Adv Healthcare Mater. 2012;1(5):537-547.

49. Chambers E, Mitragotri S. Prolonged circulation of large polymeric nanoparticles by non-covalent adsorption on erythrocytes. $J$ Control Release. 2004;100(1):111-119.

50. Domenech C, Thomas X, Chabaud S, et al. L-asparaginase loaded red blood cells in refractory or relapsing acute lymphoblastic leukaemia in children and adults: results of the GRASPALL 2005-01 randomized trial. Br J Haematol. 2011;153(1):58-65.

51. Agrawal V, Woo JH, Borthakur G, Kantarjian H, Frankel AE. Red blood cell-encapsulated L-asparaginase: potential therapy of patients with asparagine synthetase deficient acute myeloid leukemia. Protein Pept Lett. 2013;20(4):392-402. 
52. Lotero LA, Olmos G, Diez JC. Delivery to macrophages and toxic action of etoposide carried in mouse red blood cells. Biochim Biophys Acta. 2003;1620:160-166.

53. Moran NF, Bain MD, Muqit MM, Bax BE. Carrier erythrocyte entrapped thymidine phosphorylase therapy for MNGIE. Neurology. 2008;71(9):686-688.

54. Levene M, Coleman DG, Kilpatrick HC, et al. Preclinical toxicity evaluation of erythrocyte-encapsulated thymidine phosphorylase in $\mathrm{BALB} / \mathrm{c}$ mice and beagle dogs: an enzyme-replacement therapy for mitochondrial neurogastrointestinal encephalomyopathy. Toxicol Sci. 2013;131(1):311-324.

55. Kruse CA, Freehauf CL, Patel KR, Baldeschwieler JD. Mouse erythrocyte carriers osmotically loaded with methotrexate. Biotechnol Appl Biochem. 1987;9(2):123-140.

56. Biagiotti A, Rossi L, Bianchi M, et al. Immunophilin-loaded erythrocytes as a new delivery strategy for immunosuppressive drugs. J Control Release. 2011;154(3):306-313.

57. Eichler H, Gasic S, Bauer K, Korn A, Bacher S. In vivo clearance of antibody-sensitized human drug carrier erythrocytes. Clin Pharmacol Ther. 1986;40(3):300-303.

58. Del C, Batlle AM, Bustos NL, Stella AM. Enzyme replacement therapy in porphyrias - IV. First successful human clinical trial of $\delta$-aminolevulinate dehydratase-loaded erythrocyte ghosts. Int J Biochem. 1983;15(10):1261-1265.

59. Beutler E, Dale G, Guinto D, Kuhl W. Enzyme replacement therapy in Gaucher's disease: preliminary clinical trial of a new enzyme preparation. Proc Natl Acad Sci U S A. 1977;74(10):4620-4623.

60. Bax BE, Fairbanks LD, Bain MD, Simmonds HA, Chalmers RA. The entrapment of polyethylene glycol-bound adenosine deaminase (Pegademase) in human carrier erythrocytes. Biochem Soc Trans. 1996; 24(3):442-442S.

61. Hamidi M, Tajerzadeh H, Dehpour AS. Inhibition of serum angiotensinconverting enzyme in rabbits after intravenous administration of enalaprilat-loaded intact erythrocytes. J Pharm Pharmacol. 2001;53(9): 1281-1286.

62. Eichler HG, Schneider W, Raberger G, Bacher S, Pabinger I. Erythrocytes as carriers for heparin. Preliminary in vitro and animal studies. Res Exp Med. 1986;186(6):407-412.

63. Murciano JC, Medinilla S, Eslin D, Atochina E, Cines DB, Muzykantov VR. Prophylactic fibrinolysis through selective dissolution of nascent clots by tPA-carrying erythrocytes. Nat Biotechol. 2003;21(8): 891-896.

64. Annese V, Latiano A, Rossi L, et al. Erythrocytes-mediated delivery of dexamethasone in steroid-dependent IBD patients - a pilot uncontrolled study. Am J Gastroenterol. 2005;100(6):1370-1375.

65. Bossa F, Annese V, Valvano MR, et al. Erythrocytes-mediated delivery of dexamethasone 21-phosphate in steroid-dependent ulcerative colitis: a randomized, double-blind Sham-controlled study. Inflamm Bowel Dis. 2013;19(9):1872-1879.

66. Annese V, Latiano A, Rossi L. The polymorphism of multi-drug resistance 1 gene (MDR1) does not influence the pharmacokinetics of dexamethasone loaded into autologous erythrocytes of patients with inflammatory bowel disease. Eur Rev Med Pharmacol Sci. 2006; 10(1):27-31.

67. Rossi L, Serafini S, Cenerini L, et al. Erythrocyte-mediated delivery of dexamethasone in patients with chronic obstructive pulmonary disease. Biotechnol Appl Biochem. 2001;33(Pt 2):85-89.

68. Rossi L, Castro M, D’Orio F, et al. Low doses of dexamethasone constantly delivered by autologous erythrocytes slow the progression of lung disease in cystic fibrosis patients. Blood Cells Mol Dis. 2004; 33(1):57-63.

69. Chessa L, Leuzzi V, Plebani A, et al. Intra-erythrocyte infusion of dexamethasone reduces neurological symptoms in ataxia teleangiectasia patients: results of a phase 2 trial. Orphanet J Rare Dis. 2014;9:5.

70. Skorokhod OA, Kulikova EV, Galkina NM, et al. Doxorubicin pharmacokinetics in lymphoma patients treated with doxorubicin-loaded erythrocytes. Haematologica. 2007;92(4):570-571.
71. Rossi L, Pierigè F, Carducci C. Erythrocyte-mediated delivery of phenylalanine ammonia lyase for the treatment of phenylketonuria in BTBR-Pah ${ }^{\text {enu2 }}$ mice. J Control Release. 2014;194:37-44.

72. Godfrin Y, Horand F, Franco R, et al. Meeting highlights: international seminar on the red blood cells as vehicles for drugs. Expert Opin Biol Ther. 2012;12(1):127-133.

73. Kosenko EA, Venediktova NI, Kudryavtsev AA, et al. Encapsulation of glutamine synthetase in mouse erythrocytes: a new procedure for ammonia detoxification. Biochem Cell Biol. 2008;86(6):469-476.

74. Sinauridze EI, Vuimo TA, Kulikova EV, Shmyrev II, Ataullakhanov FI. A new drug form of blood coagulation factor IX: red blood cellentrapped factor IX. Med Sci Monit. 2010;16(10):19-26.

75. Bourgeaux V, Hequet O, Campion Y, et al. Inositol hexaphosphateloaded red blood cells prevent in vitro sickling. Transfusion. 2010;50(10): 2176-2184.

76. Pierigè $\mathrm{F}$, Serafini $\mathrm{S}$, Rossi L, Magnani M. Cell-based drug delivery. Adv Drug Deliv Rev. 2008;60(2):286-295.

77. Magnani M, Chiarantini L, Vittoria E, Mancini U, Rossi L, Fazi A. Red blood cells as an antigen-delivery system. Biotechnol Appl Biochem. 1992;16(2):188-194.

78. Kwon YM, Chung HS, Moon C, et al. L-Asparaginase encapsulated intact erythrocytes for treatment of zcute lymphoblastic leukemia (ALL). J Control Release. 2009;139:182-189.

79. Zarrin A, Foroozesh M, Hamidi M. Carrier erythrocytes: recent advances, present status, current trends and future horizons. Expert Opin Drug Deliv. 2014;11(3):433-447.

80. Mura S, Couvreur P. Nanotheranostics for personalized medicine. Adv Drug Deliv Rev. 2012;64(13):1394-1416.

81. Wong AK. Platelet biology: the role of shear. Expert Rev Hematol. 2013;6(2):205-212.

82. Walsh TG, Metharom P, Berndt MC. The functional role of platelets in the regulation of angiogenesis. Platelets. 2015;26(3):199-211.

83. Münzer $\mathrm{P}$, Borst $\mathrm{O}$, Walker $\mathrm{B}$, et al. Acid sphingomyelinase regulates platelet cell membrane scrambling, secretion, and thrombus formation. Arterioscler Thromb Vasc Biol. 2014;34(1):61-71.

84. Shi QZ, Montgomery RR. Platelets as delivery systems for disease treatments. Adv Drug Deliv Rev. 2010;62(12):1196-1203.

85. Geraldo RB, Sathler PC, Lourenco AL, et al. Platelets: still a therapeutical target for haemostatic disorders. Int J Mol Sci. 2014;15(10): 17901-17919.

86. White JG. Platelets are covercytes, not phagocytes: uptake of bacteria involves channels of the open canalicular system. Platelets. 2005; 16(2):121-131.

87. Riess L. Zurpathologischenanatomie des blutes [The pathological anatomy of the blood]. Arch Anat Physiol Wissensch Med. 1872;39: 237-249. German.

88. Billroth T. Lectures on Surgical Pathology and Therapeutics: A Handbook for Students and Practitioners. London: The New Sydenham Society; 1878.

89. Buergy D, Wenz F, Groden C, Brockmann MA. Tumor-platelet in interaction in solid tumors. Int J Cancer. 2012;130(12):2747-2760.

90. Bambace NM, Holmes CE. The platelet contribution to cancer progression. J Thromb Haemost. 2011;9(2):237-249.

91. Gasic GJ, Gasic TB, Stewart CC. Antimetastatic effects associated with platelet reduction. Proc Natl Acad Sci U S A. 1968;61(1):46-52.

92. Sarkar S, Alam MA, Shaw J, Dasgupta AK. Drug delivery using platelet cancer cell interaction. Pharm Res. 2013;30(11):2785-2794.

93. Greineder CF, Howard MD, Carnemolla R, Cines DB, Muzykantov VR. Advanced drug delivery systems for antithrombotic agents. Blood. 2013;122(9):1565-1575.

94. Chen H, Mo W, Su H, Zhang Y, Song H. Characterization of a novel bifunctional mutant of staphylokinase with platelet-targeted thrombolysis and antiplatelet aggregation activities. BMC Mol Biol. 2007;8:88.

95. Davies A, Lewis DJ, Watson SP, Thomas SG, Pikramenou Z. $\mathrm{pH}$-controlled delivery of luminescent europium coated nanoparticles into platelets. Proc Natl Acad Sci U S A. 2012;109(6):1862-1867. 
96. Aurich K, Spoerl MS, Furll B, et al. Development of a method for magnetic labeling of platelets. Nanomedicine. 2012;8(5):537-544.

97. Kona S, Dong JF, Liu YL, Tan J, Nguyen KT. Biodegradable nanoparticles mimicking platelet binding as a targeted and controlled drug delivery system. Int J Pharm. 2012;423(2):516-524.

98. Lin A, Sabnis A, Kona S, et al. Shear-regulated uptake of nanoparticles by endothelial cells and development of endothelial-targeting nanoparticles. J Biomed Mater Res A. 2009;93(3):833-842.

99. Modery-Pawlowski CL, Master AM, Pan V, Howard GP, Sen Gupta A. A platelet-mimetic paradigm for metastasis-targeted nanomedicine platforms. Biomacromolecules. 2013;14(3):910-919.

100. Hu CM, Fang RH, Wang KC, et al. Nanoparticle biointerfacing by platelet membrane cloaking. Nature. 2015;526(7571):118-121.

101. Shi Q, Fahs SA, Wilcox DA, et al. Syngeneic transplantation of hematopoietic stem cells that are genetically modified to express factor VIII in platelets restores hemostasis to hemophilia A mice with preexisting FVIII immunity. Blood. 2008;112(7):2713-2721.

102. Buckley MF, James JW, Brown DE, et al. A novel approach to the assessment of variations in the human platelet count. Thromb Haemost. 2000;83(3):480-484.

103. Bern M, Sand MK, Nilsen J, Sandlie I, Andersen JT. The role of albumin receptors in regulation of albumin homeostasis: implications for drug delivery. J Control Release. 2015;211:144-162.

104. Kratz F. Albumin as a drug carrier: design of prodrugs, drug conjugates and nanoparticles. J Control Release. 2008;132(3):171-183.

105. Babson AL, Winnick T. Protein transfer in tumor-bearing rats. Cancer Res. 1954;14(8):606-611.

106. Stehle G, Sinn H, Wunder A, et al. Plasma protein (albumin) catabolism by the tumor itself - implications for tumor metabolism and the genesis of cachexia. Crit Rev Oncol Hematol. 1997;26(2): $77-100$.

107. Wunder A, Muller-Ladner U, Stelzer EH, et al. Albumin-based drug delivery as novel therapeutic approach for rheumatoid arthritis. J Immunol. 2003;170(9):4793-4801.

108. Burger AM, Hartung G, Stehle G, Sinn H, Fiebig HH. Pre-clinical evaluation of a methotrexate-albumin conjugate (MTX-HSA) in human tumor xenografts in vivo. Int J Cancer. 2001;92(5):718-724.

109. Bolling C, Graefe T, Lubbing C, et al. Phase II study of MTX-HSA in combination with cisplatin as first line treatment in patients with advanced or metastatic transitional cell carcinoma. Invest New Drugs. 2006;24(6):521-527.

110. Teneriello MG, Tseng PC, Crozier M, et al. Phase II evaluation of nanoparticle albumin-bound paclitaxel in platinum-sensitive patients with recurrent ovarian, peritoneal, or fallopian tube cancer. $J$ Clin Oncol. 2009;27(9):1426-1431.

111. Yuan ZX, He XK, Wu XJ, et al. Peptide fragments of human serum albumin as novel renal targeting carriers. Int J Pharm. 2014; 460(1-2):196-204.

112. Han JH, Wang Q, Zhang ZR, Gong T, Sun X. Cationic bovine serum albumin based self-assembled nanoparticles as siRNA delivery vector for treating lung metastatic cancer. Small. 2014;10(3):524-535.

113. Asmatulu R, Yang SY, Abedin F, Anwar MD. Albumin-based microcomposite drug carriers with dual chemo-agents for targeted breast cancer treatment. J Biomater Appl. 2015;30(1):38-49.

114. Peralta D, Heidari Z, Dash S, Tarr MA. Hybrid paclitaxel and gold nanorod-loaded human serum albumin nanoparticles for simultaneous chemotherapeutic and photothermal therapy on $4 \mathrm{~T} 1$ breast cancer cells. ACS Appl Mater Interfaces. 2015;7(13):7101-7111.

115. Zhang Y, Ho A, Yue JP, et al. Structural basis and anticancer properties of ruthenium-based drug complexed with human serum albumin Eur J Med Chem. 2014;86:449-455.

116. Volk LD, Flister MJ, Chihade D, Desai N, Trieu V, Ran S. Synergy of nab-paclitaxel and bevacizumab in eradicating large orthotopic breast tumors and preexisting metastases. Neoplasia. 2011;13(4):327-338.

117. Elzoghby AO, Samy WM, Elgindy NA. Albumin-based nanoparticles as potential controlled release drug delivery systems. J Control Release. 2012;157(2):168-182.
118. Kamalinia G, Khodagholi F, Shaerzadeh F, et al. Cationic albuminconjugated chelating agent as a novel brain drug delivery system in neurodegeneration. Chem Biol Drug Des. 2015;86(5):1203-1214.

119. Sebak S, Mirzaei M, Malhotra M, Kulamarva A, Prakash S. Human serum albumin nanoparticles as an efficient noscapine drug delivery system for potential use in breast cancer: preparation and in vitro analysis. Int J Nanomedicine. 2010;5:525-532.

120. Thao le Q, Byeon HJ, Lee C, et al. Pharmaceutical potential of tacrolimus-loaded albumin nanoparticles having targetability to rheumatoid arthritis tissues. Int J Pharm. 2016;497(1-2):268-276.

121. Kuipers ME, Swart PJ, Witvrouw M, et al. Anti-HIV-1 activity of combinations and covalent conjugates of negatively charged human serum albumins (NCAs) and AZT. J Drug Targeting. 1999;6(5): 323-335.

122. Kuipers ME, Berg M, Swart PJ, et al. Mechanism of anti-HIV activity of succinylated human serum albumin. Biochem Pharmacol. 1999; 57(8):889-898.

123. Kim I, Kim TH, Ma K, et al. Synthesis and evaluation of human serum albumin-modified exendin-4 conjugate via heterobifunctional polyethylene glycol linkage with protracted hypoglycemic efficacy. Bioconjug Chem. 2010;21(8):1513-1519.

124. Das S, Banerjee R, Bellare J. Aspirin loaded albumin nanoparticles by coacervation: implications in drug delivery. Trends Biomater Artif Organs. 2005;18(2):203-212.

125. Kufleitner J, Wagner S, Worek F, Briesen H, Kreuter J. Adsorption of obidoxime onto human serum albumin nanoparticles: drug loading, particle size and drug release. J Microencapsul. 2010;27(6): 506-513.

126. Kufleitner J, Worek F, Kreuter J. Incorporation of obidoxime into human serum albumin nanoparticles: optimisation of preparation parameters for the development of a stable formulation. $J$ Microencapsul. 2010;27(7):594-601.

127. Graeser R, Esser N, Unger H. INNO-206, the (6-maleimidocaproyl hydrazone derivative of doxorubicin), shows superior antitumor efficacy compared to doxorubicin in different tumor xenograft models and in an orthotopic pancreas carcinoma model. Invest New Drugs. 2010; 28(1):14-19.

128. Sleep D. Albumin and its application in drug delivery. Expert Opin Drug Deliv. 2015;12(5):793-812.

129. Yang Z, Wang X, Diao H, et al. Encapsulation of platinum anticancer drugs by apoferritin. Chem Commun (Camb). 2007;7(33): 3453-3455.

130. Huang Y, Wang YJ, Wang Y, et al. Exploring naturally occurring ivy nanoparticles as an alternative biomaterial. Acta Biomater. 2015; 25:268-283.

131. Wang YJ, Huang YJ, Anreddy N. Tea nanoparticle, a safe and biocompatible nanocarrier, greatly potentiates the anticancer activity of doxorubicin. Oncotarget. 2015;7(5):5877-5891.

132. Yi S, Wang Y, Huang Y, et al. Tea nanoparticles for immunostimulation and chemo-drug delivery in cancer treatment. J Biomed Nanotechnol. 2014;10(6):1016-1029.

133. Wang Y, Yi S, Sun L, Huang Y, Zhang M. Charge-selective fractions of naturally occurring nanoparticles as bioactive nanocarriers for cancer therapy. Acta Biomater. 2014;10(10):4269-4284.

134. Jin H, Lovell JF, Chen J, et al. Mechanistic insights into LDL nanoparticle-mediated siRNA delivery. Bioconjug Chem. 2011;23(1): 33-41.

135. Watterlot L, Rochat $\mathrm{T}$, Sokol H, et al. Intragastric administration of a superoxide dismutase-producing recombinant Lactobacillus casei BL23 strain attenuates DSS colitis in mice. Int J Food Microbiol. 2010;144(1):35-41.

136. Lautenschlager C, Schmidt C, Fischer D, Stallmach A. Drug delivery strategies in the therapy of inflammatory bowel disease. Adv Drug Deliv Rev. 2014;71:58-76.

137. Gutierrez Millan C, Colino Gandarillas CI, Sayalero Marinero ML, Lanao JM. Cell-based drug-delivery platforms. Ther Deliv. 2012;3(1): 25-41. 


\section{Publish your work in this journal}

OncoTargets and Therapy is an international, peer-reviewed, open access journal focusing on the pathological basis of all cancers, potential targets for therapy and treatment protocols employed to improve the management of cancer patients. The journal also focuses on the impact of management programs and new therapeutic agents and protocols on

patient perspectives such as quality of life, adherence and satisfaction. The manuscript management system is completely online and includes a very quick and fair peer-review system, which is all easy to use. Visit http://www.dovepress.com/testimonials.php to read real quotes from published authors.

Submit your manuscript here: http://www.dovepress.com/oncotargets-and-therapy-journal 